\title{
Remote Sensing of Cell-Culture Assays
}

\author{
Pablo Pérez, Andrés Maldonado-Jacobi, \\ Antonio J. López, Cristina Martínez, Alberto Olmo, \\ Gloria Huertas and Alberto Yúfera
}

Additional information is available at the end of the chapter

http://dx.doi.org/10.5772/67496

\begin{abstract}
This chapter describes a full system developed to perform the remote sensing of cellculture experiments from any access point with internet connection. The proposed system allows the real-time monitoring of cell assays thanks to bioimpedance measurement circuits developed to count the number of cell present in a culture. Cell-culture characterization is performed through the measurement of the increasing bioimpedance parameter over time. The circuit implementation is based on the oscillation-based test (OBT) methodology. Bioimpedance of cell cultures is measured in terms of the oscillation parameters (frequency, amplitude, phase, etc.) and used as empirical markers to carry out an appropriate interpretation in terms of cell size identification, cell counting, cell growth, growth rhythm, etc. The device is capable of managing the whole sensing task and performs wireless communication through a Bluetooth module. Data are interpreted and displayed on a computer or a mobile phone through a web application. The system has its practical application in drug development processes, offering a label-free, high-throughput, and high-content screening method for cellular research, avoiding the classical end-point techniques and a significant workload and cost material reduction.
\end{abstract}

Keywords: bioimpedance, ECIS, cell-culture assay, microelectrode, OBT

\section{Introduction and motivation}

The electrical impedance of a biological material reflects the actual physical properties of a biosample. In frequency-dependent analysis, the $\beta$-dispersion ranging from kilohertz to hundreds of megahertz [1-3] is mainly affected by the shape of the cells, the structure of the 
cell membranes, the amount of intra- and extracellular water, etc., enabling the possibility to be used as biomarker in cell-culture test. From this basis, the electrical bioimpedance (BI) is employed to assess properties of biological materials, such as cancer cells [4, 5]; since healthy and cancer cells are different in shape, size, and performance, cancer cells could be detected by using their impedance as marker. Among impedance spectroscopy (IS) techniques, electrical cell impedance spectroscopy (ECIS) [6, 7], based on two electrode setups, allows the measurement of cell-culture impedances, the definition of the biological nature-material, internal activity, and size-of a cell line, and its relationship with the environment (transfer flow through the cell membrane) [8]. In addition, ECIS technique can be used in other cellculture assays such as toxicology [9, 10], motility [11], cell growth, and cell line identification [12], based on their bioimpedance test.

ECIS technique relies on appropriate electrical models to decode the electrical response produced by the stimuli. To manage proficiently bioimpedance data, confident electrical models of the full system composed by the electrodes and cells are required. Several works have been developed in this area [7,13-17]. These models are the key for matching electrical simulations to real system performance and hence decoding correctly the results obtained from experiments. This problem is usually referred to as a reconstruction problem.

Furthermore, accurate measurement techniques and circuits are compulsory for bioimpedance tests. In these techniques, different measurement configurations are described varying between two and four electrode setups. Such configuration depends on the specifications of the problem to be solved [18, 19]. Good electrical modeling of electrodes and biosamples is mandatory for a correct circuit design and the data measurement decoding [9].

Finally, the technique enables biomedical researchers and lab technicians to real-time monitor cell-culture assays [20]. This fact represents not only the possibility of continuously performed cell-culture assay supervision, avoiding the classical end-point techniques, but also a significant workload and cost material reduction. The ECIS method only requires one culture to perform the full assay and not many samples, as commonly, for time supervision and characterization of the assays. Even more, it enables a real-time remote sensing option for the biomedical assays, in the context of the so-called topic of internet of things (IoT), by using wireless communication protocols and electronic devices for internet access, as laptops and cellular phones. This approach to new concept of cell-culture assays allows biomedical researchers to supervise a culture in any time from any place, making it easy and efficient for lab protocol implementation.

This chapter presents, at first, a full system for bioimpedance measurements of cell-culture experiments [21], useful at biomedical labs, that our team has developed for remote monitoring of assays. Our objectives are, firstly, to reduce the work task at biomedical labs; secondly, to look forward to derive accurate algorithms and techniques for bioimpedance tests, applied to cellculture assays; and, finally, to make the test easy by delivering the possibility of remote sensing of experiments through wireless communication. The conceptual diagram of the proposed system is shown in Figure 1(a). The circuits for test are introduced into the incubator chamber, together with the cell culture. Once the test has been performed and the electrical relevant information about required biomarkers is acquired, the measurement data from assay evolution are 


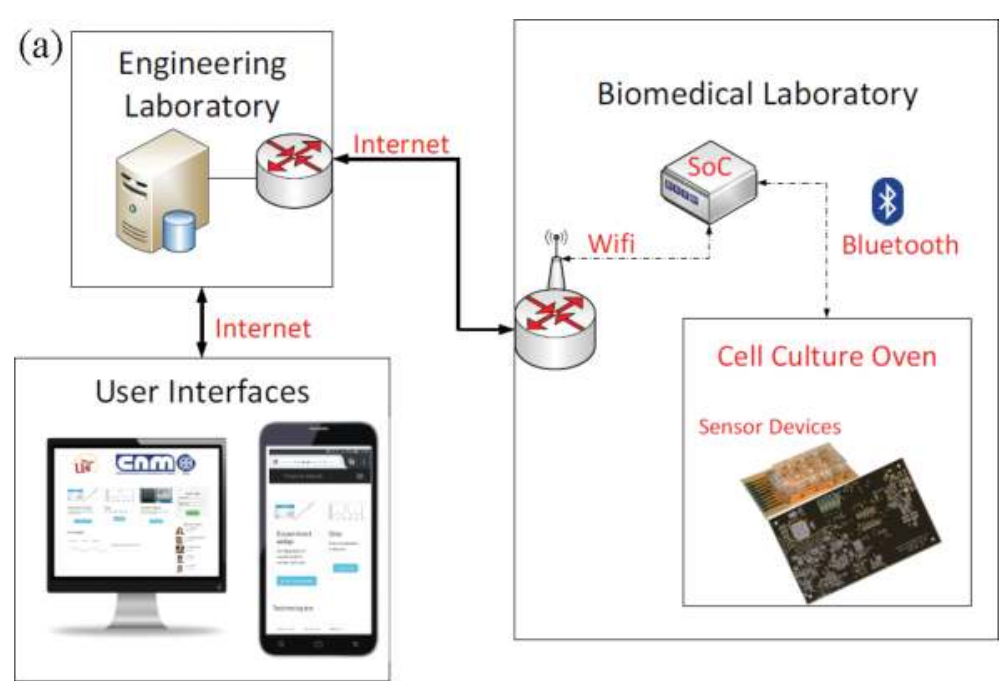

(b)

Frequency vs Time

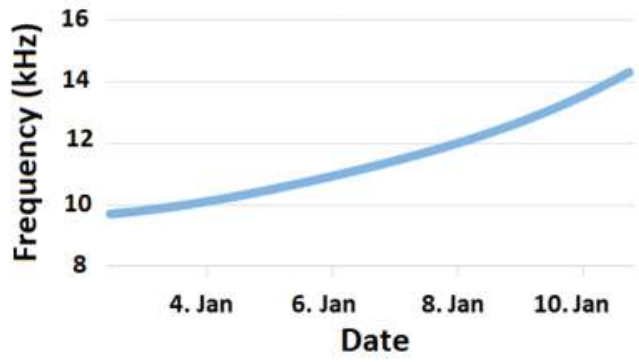

Amplitude vs Time

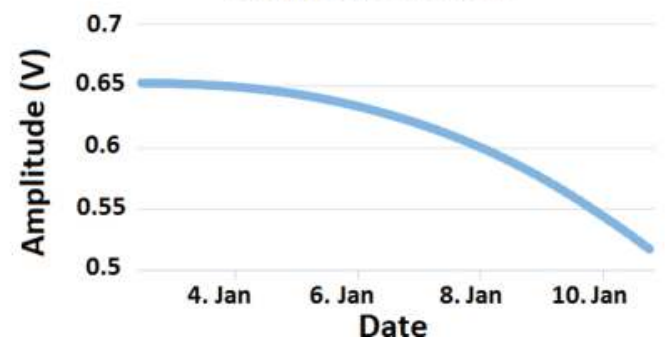

Figure 1. Proposed system for cell-culture test: (a) Block diagram. Bioimpedance data are measured at the incubator chamber by the proposed circuits and then sent to a computer server. (b) Frequency and voltage amplitude, parameters expected from test, as a function of the cell-culture evolution. Increasing time means more cells at the culture and increasing impedance values. Frequency and voltage amplitude will change with the bioimpedance evolution.

sent from $\mu \mathrm{P}$ circuit to external wireless emitter (Linux-based system on a chip (SoC)), which is responsible to store this information over the internet. Data are managed and packed on a database in a data server, from which are available to researches, from the laptop or phone in 
real time. Figure 1(b) shows a typical graphic obtained for a typical cell-culture test example, in which the electrical variables (oscillation frequency or amplitude ${ }^{1}$ ) are displayed in time, 15 min each, giving information of how the cells in the culture are evolving. In this case, increasing values of frequency mean increasing cell population. To know exactly the number of cells, this information must be adequately decoded, by employing electrical models for the cell-electrode system, such as we will explain in the chapter.

\section{Electronic system description}

The full system presented for remote sensing of cell-culture assays has four functional parts: (1) the bioimpedance (BI) sensor to evaluate the electrical response. It is possible to connect that response with the current cell number and even use it as a biomarker for characterization of different cell lines, (2) the BI algorithm employed to obtain the magnitude and/or phase of the BI under test, (3) the measurement circuits that physically will implement the algorithm to electrical signals, and (4) the communication system and server functionality for remote system access for users.

\subsection{Sensor and cell-electrode model}

To implement the ECIS technique, two electrodes are needed, the sensing and the GND electrodes, which are excited by an AC current source $\left(i_{x}\right)$ at several frequencies. The impedance $Z_{x}$ (magnitude and phase) is obtained by measuring the voltage response $\left(v_{x}\right)$ in the signal path, from one electrode to the other through the cell-culture system, for each frequency of interest. Figure 2 shows an example of ECIS electrode employed for the experiments from Applied Biophysics (www.biophysics.com). Cell adhesion to the bottom surface will derive on impedance changes, which value will increase with the number of cells attached to such the bottom surface of the electrodes. To characterize these impedance measurements, the electrode material and geometry, the electrical cell-electrode model [9], and the working frequency must be known.

The concept described here employs 8W10E electrodes, each one containing 8 wells. Our aim is to reduce the required circuitry for impedance measurements [18], avoiding the need of any input stimuli from the outside [22]. Here, we apply the so-called oscillation-based (OB) method $[23,24]$ to improve and simplify the test, by transforming the cell-culture under test (CCUT) into an oscillator. For that, only a few extra external components to force the "biological circuit" to oscillate are required. All variations inside the cell-culture under test in time (increasing number of cell) will provoke modifications on the frequency/amplitude of the voltage oscillations and consequently being these alterations observable. As the main advantages of this technique, the need of complex resources for stimuli generation is avoided and means a simplification in the number of circuits for test $[25,26]$.

${ }^{1}$ In this case instead of oscillation amplitude, the variable being displayed is the automatic gain controller (AGC). See Section 2.3 for further detail. 

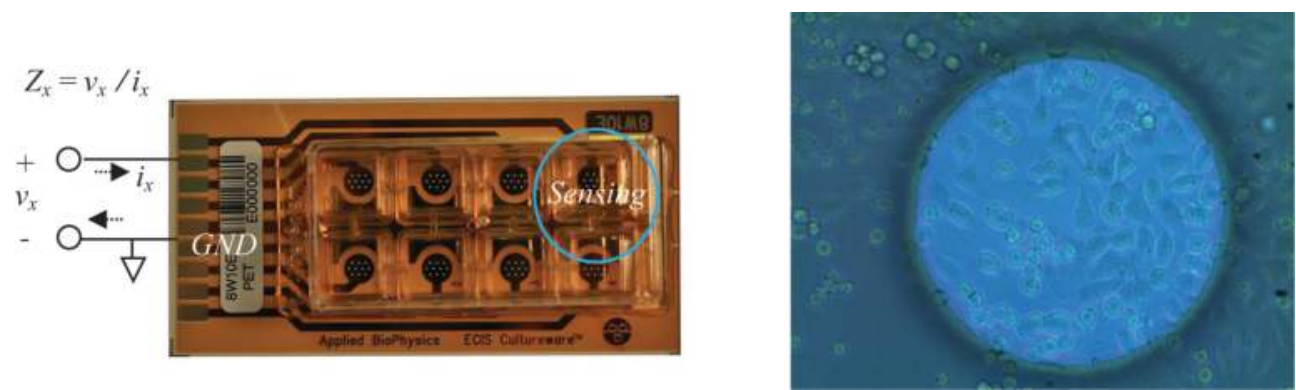

Figure 2. The $8 \mathrm{E} 10 \mathrm{~W}$ system. Each system has 8 separated wells for cell culture and 10 electrodes of $250 \mu \mathrm{m}$ diameter by well (www.biophysics.com). On the right side, an electrode with the Chinese hamster ovary fibroblast cell line, AA8 (American Type Culture Collection), cultured over the surface of one circular electrode can be seen.

The cell-electrode sensor: The impedance under test of a two-electrode system like [27] has several circuit elements for each electrode. For an electrode in saline solution, we have to consider the double-layer capacitance $\left(C_{d l}\right)$ and the transfer resistance $\left(R_{p}\right)$ in parallel. The parallel connection of $C_{d l}$ and $R_{p}$ is called $Z(\omega)$ in Figure 3(b). Also, there is the spreading resistance $\left(R_{s}\right)$ for electrodes non-covered by cells, which is in series with $Z(\omega)$. All these four circuit elements are described in Figure 3(b). The parameter $A$ represents the sensing electrode surface where the cell-culture growths. Cells are usually attached to the electrode surface,

(a)

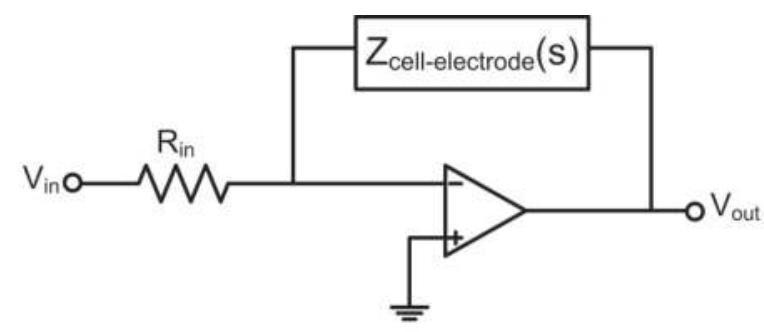

(b)

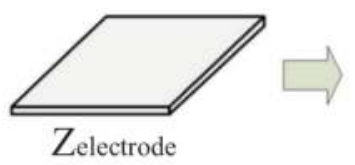

(c)

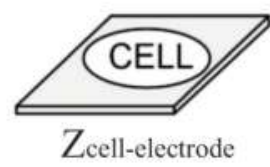

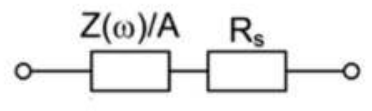

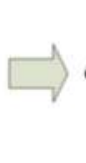

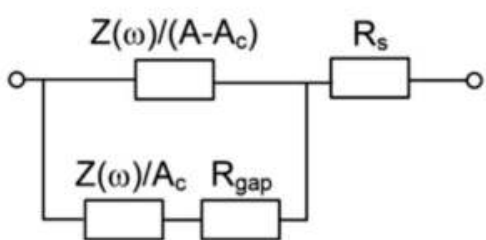

Figure 3. (a) Circuit proposed for cell-electrode impedance testing. (b) Circuit model for a bare electrode of area A. (c) Circuit model for an electrode of area A, partially covered with cells with an area A. Estimated values for a $50 \times 50 \mu \mathrm{m}^{2}$ gold electrode: $\mathrm{R}_{\mathrm{s}}=5.4 \mathrm{k} \Omega, \mathrm{Z}(\omega)=\mathrm{C}_{\mathrm{dl}}|| \mathrm{R}_{\mathrm{p}^{\prime}}$ with $\mathrm{C}_{\mathrm{dl}}=0.37 \mathrm{nF}$ and $\mathrm{R}_{\mathrm{p}}=25 \mathrm{M} \Omega . \mathrm{R}_{\text {gap }}=75 \mathrm{k} \Omega$. 
covering a given area $A_{c}$. More cells mean increasing values of $A_{c}$ parameter. A new circuit element appears the gap resistance $\left(R_{g a p}\right)$, to model this cell attachment effect [13,14]. Our work employs circular gold microelectrodes of $250 \mu \mathrm{m}$ diameter, which can be totally or partially covered by cells in the culture. The fill factor (ff) parameter as the percent of the electrode area $(A)$ covered by cells $\left(A_{c}\right)$, being always less than one, has been defined.

The setup shown in Figure 3 proposes an experimental circuit, including the circuit model for the electrode, to study the sensor impedance for a given electrode in which part of its surface, is covered by cells, $Z_{\text {cell-electrode }}(s)$. The purpose of the $R_{\text {in }}$ resistor is to limit the current flowing across the cell-electrode in adequate signal levels (below $20 \mu \mathrm{A}$ for cell protection). In fact, circuit in Figure 3(a) works as an inverting voltage amplifier, converting the input voltage in an amplified version at the output voltage, which must be below to $50-100 \mathrm{mV}$ for electrode modeling constrains [14]. The Bode plot (magnitude and phase) for this bioelectronic system $H_{z}(s)$ in Figure 4 shows curves for several cell occupation areas, ff. Let us notice that, in this case, when frequencies around $8 \mathrm{kHz}$ are considered, the system has optimum ff sensitivity. This means that both, magnitude and phase response, can be correlated to the fill factor parameter or cell-to-electrode area overlap, $A_{c}$. Most ECIS techniques search for the best frequency response for optimum impedance characterization and then perform the measurements knowing the ff dependence. Absolute magnitudes (Figure 4) or normalized magnitudes of cell index (CI) can be used as sensitivity curves for this impedance sensors and assays. We will use the ff-dependent magnitude and phase curves of the bioelectrical model for $H_{z}(s)=-Z_{\text {cell-electrode }}(s) / R_{\text {in' }}$ in Figures 3 and 4 , to be employed as part of a more complex circuit to force an oscillatory behavior, directly correlated with the cell-to-electrode area overlap.

In order to test our sensor sensitivity, we have proposed a set of experiments using the $8 \mathrm{~W} 10 \mathrm{E}$ electrode system and the cell line U2OS (isolated from a mild osteosarcoma in 1964). At the

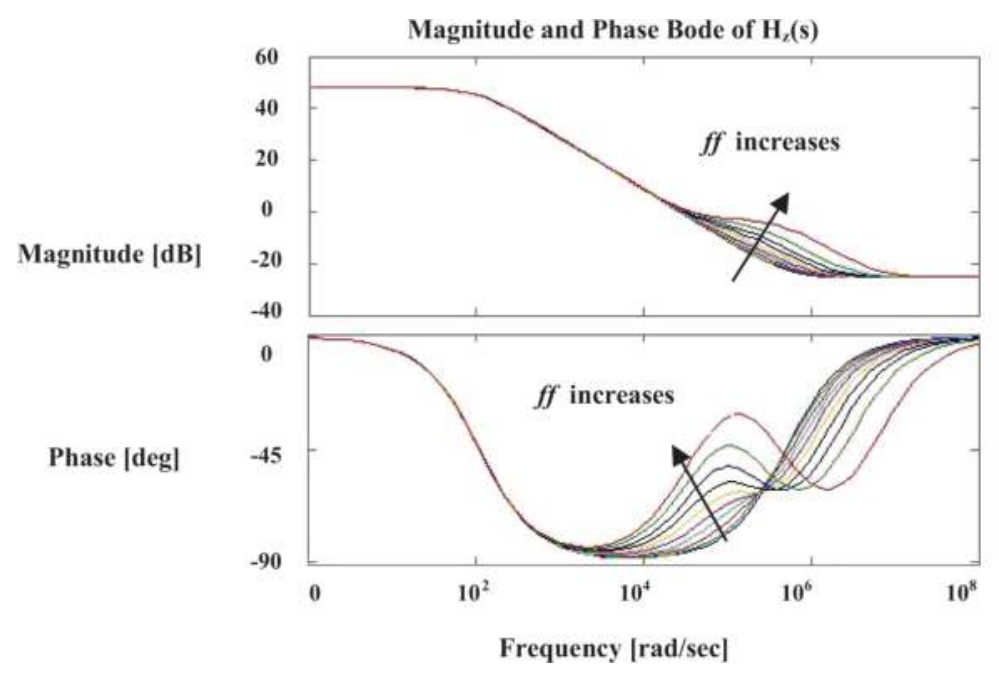

Figure 4. Bioelectronic system Bode curves for $H_{z}(s)=-Z_{\text {cell-electrode }} / R_{i n}$ using several cell-culture occupation areas (ff). 
chromosomal level, its karyotype is greatly altered, with a ploidy in the range of hypertriploid. These cells, in culture, grow as an adherent monolayer and, when seeded at low density, have a tendency to grow forming discrete colonies. The objective of these assays was to evaluate the sensitivity of the ECIS sensor. For that, the eight wells were seeded with (a) only medium ( 2 wells), (b) 500 cells ( 2 wells), (c) 1000 cells ( 2 wells), and (d) 2000 cells ( 2 wells). Figure 5 shows the image of the cell cultures after a 5-day growth. The experimental transfer function $H_{z}(s)$ (Figure 3) was measured (magnitude and phase) using a network analyzer. The phase response is shown in Figure 6, illustrating how at $8 \mathrm{kHz}$, the number of cells (fill-factor) can be estimated by measuring that parameter (the phase response) at $V_{\text {out }}$ (see Figure 3). Table 1 summarizes the experimental data at $8 \mathrm{kHz}$, achieving a sensor sensitivity as expected, showing that the magnitude and phase correlate with the cell population.

We are currently also considering the possibility of employing an electrode as reference at the inverter amplifier, as it is represented at Figure 7. This configuration employs the reference electrode to normalize the output voltage of the impedance converter, in such a way that inverter amplifier voltage gain $\left(V_{\text {out }} / V_{\text {out } 2}\right)$ will have to gain 1 , when both wells are emptied, and will increase its absolute value for increasing values when cells are seeded at sensing electrode and then start to grow. This performance is represented in Figure 7(b).

\subsection{Bioimpedance technique}

Previously in this chapter, the idea of the proposed measurement system was presented. This system requires the conversion of the cell-culture under test into a robust oscillator, by adding some extra components. Figure 8 shows the block components to perform the proposed transformation. In order to force oscillations, a positive feedback loop has to be added. For OB application, the accurate prediction of the oscillation parameters (frequency and amplitude), by means of theoretical analysis or programming the suitable simulations, is mandatory [28].

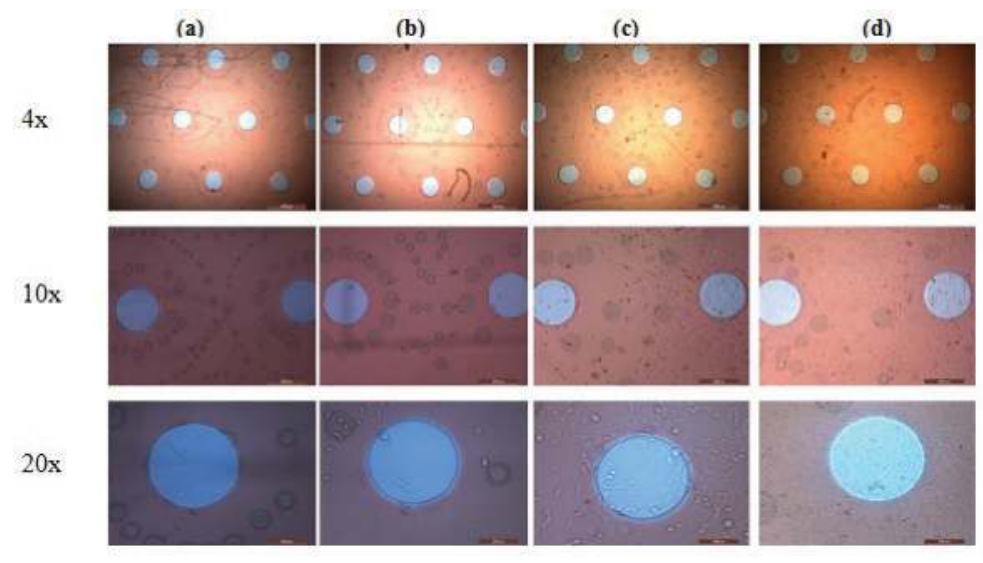

Figure 5. Cell-culture images showing four wells with (a) only medium, (b) 500 initial cells, (c) 1000 initial cells, and (d) 2000 initial cells, at different zooms. 


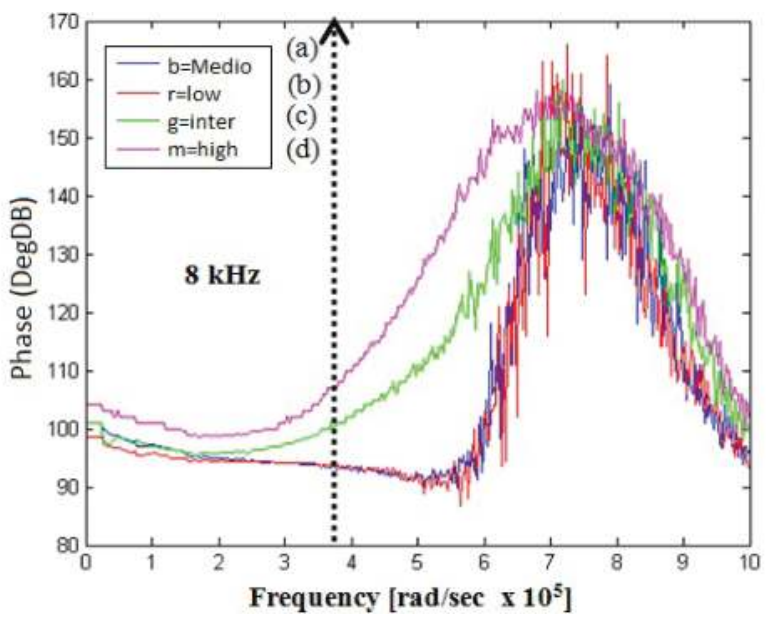

Figure 6. Experimental phase response obtained for (a) medium, (b) 500 cells assay, (c) 1000 cells assays, and (d) 2000 cells assays, after 5 days of growth.

\begin{tabular}{lll}
\hline Cells at time $=0$ & Magnitude & Phase (deg.) \\
\hline Medium (no cells) & $-68 \mathrm{~dB}$ & 90 \\
Low (500 cells) & $-67 \mathrm{~dB}$ & 93 \\
Int (1000 cells) & $-66 \mathrm{~dB}$ & 108 \\
High (2000 cells) & $-61 \mathrm{~dB}$ & 140 \\
\hline
\end{tabular}

Table 1. Magnitude and phase response at $8 \mathrm{kHz}$ (5 days of growth).

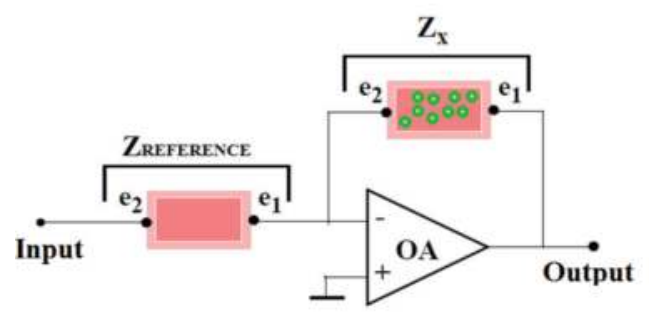

(a)

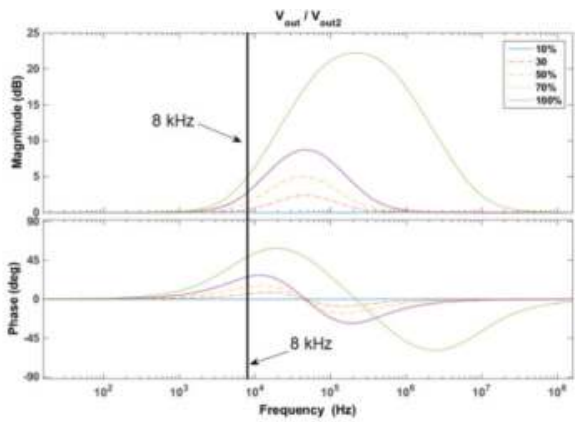

(b)

Figure 7. (a) Block diagram proposed system setup with a reference electrode. (b) Bode graphics for the magnitude and phase frequency response of the electrode and cell-culture model using the reference electrode $Z_{\text {reference }}$ and $V_{\text {out }} / V_{\text {out } 2}$ in Figure 3. Both curves can be considered as sensitivity curves for the bioimpedance sensor, being the magnitude one similar to the so-called cell index in cell-culture assays. 


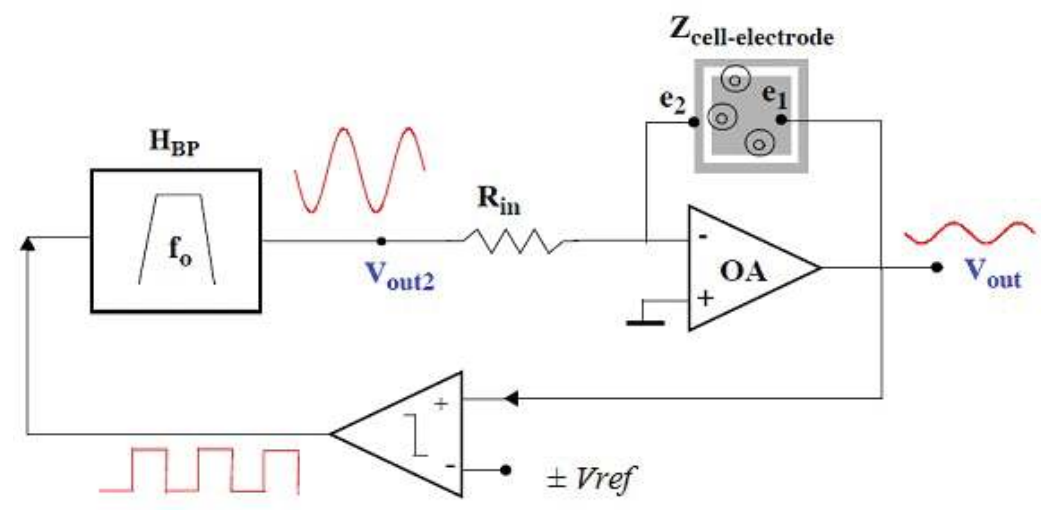

Figure 8. Block diagram of the proposed OB implementation. Oscillations $\left(a_{o s c} f_{o s c}\right)$ will be measured at $V_{\text {out } 2}$ signal.

A nonlinear element (a simple comparator) within the feedback loop in Figure 7 is added to avoid the saturation of the active elements and then to keep sustained oscillations [29]. The oscillation amplitudes are controlled with precision thanks to this element. A set of oscillation conditions can be fulfilled to guarantee that the oscillations are alive. One of the simplest ways to implement the oscillator includes a band-pass filter (BPF) in the loop, as proposed in Figure 7.

In this work, the abovementioned scheme (Figure 8) to implement the oscillator is proposed. The nonlinear feedback element and the "biological filter" are connected to implement the oscillator. In this way, only the input and output of such a "biological filter" are manipulated to perform the test allowing a low intrusion in the structure.

Let us now consider the case of a comparator with saturation levels $\pm V_{\text {ref }}$ and a second-order band-pass filter (BPF). This closed-loop system verifies the required properties: the system is autonomous, the linear transfer function contains enough low-pass filtering to neglect the higher harmonics at the comparator output, and the nonlinearity is separable and frequency independent.

An oscillatory solution for the first-order describing function (DF) equation of the nonlinear block in the closed-loop system in Figure 8, $N(a)+1 / H(s)=0$, can be forced by choosing adequately the BPF. In the solution, the oscillatory parameters are $\left(\omega_{\text {osc }} a_{\text {osc }}\right)$, being $\omega_{\text {osc }}$ and $a_{\text {osc }}$ the frequency and amplitude of the oscillation, respectively. The DF function in this case is $N(a)$, whereas $H(s)$ is the closed-loop system transfer function. For modeling the system, the characteristic equation can be set as

$$
1+N(a) \cdot H(s)=0
$$

where $N(a)$ is the comparator DF and $H(s)$ the modified system: the band-pass filter plus the bioimpedance transfer function. The general BPF transfer function is given by 


$$
H_{B P}(s)=\frac{k_{1}^{*} \frac{\omega_{o}^{*}}{Q^{*}} s}{s^{2}+\frac{\omega_{o}^{*}}{Q^{*}} s+\omega_{o}^{* 2}}
$$

And the corresponding bioimpedance sensor transfer function, $H_{z}(s)$, is

$$
H_{Z}(s)=\frac{k_{2} s^{2}+k_{1} \frac{\omega_{o}}{Q} s+k_{o} \omega_{o}^{2}}{s^{2}+\frac{\omega_{o}}{Q} s+\omega_{o}^{2}}
$$

being the constant parameters $\left(\omega_{0^{\prime}}, Q\right.$; and $k_{o^{\prime}} k_{1}$, and $\left.k_{2}\right)$ directly related to the electrode size, technology, and biological material (ff). Then, the global function expression will be given by

$$
H(s)=H_{B P}(s) \cdot H_{Z}(s)
$$

To force the oscillations, a pair of complex poles of the overall system has to be placed on the imaginary axes. The way to determine the oscillation conditions (gain, frequency, and amplitude) is solving Eq. (1). This is equivalent to find the solution of this equation set:

$$
1+N(a) H(s)=\left(s^{2}+w_{o s c}^{2}\right)\left(s^{2}+B s+A\right)=0
$$

where the coefficients are obtained from the equation system:

$$
\begin{aligned}
& B=\frac{w_{o}}{Q}+\frac{w_{o}^{*}}{Q^{*}}+N\left(a_{o s c}\right) k_{1}^{*} k_{2} \frac{w_{o}^{*}}{Q^{*}} \\
& A+w_{o s c}^{2}=w_{o}^{2}+\frac{w_{o}}{Q} \frac{w_{o}^{*}}{Q^{*}}+w_{o}^{* 2}+N\left(a_{o s c}\right) k_{1}^{*} \frac{w_{o}^{*}}{Q^{*}} k_{1} \frac{w_{o}}{Q} \\
& B \times w_{o s c}=\frac{w_{o}^{*}}{Q^{*}} w_{o}^{2}+\frac{w_{o}^{*}}{Q^{*}} w_{o}^{* 2}+N\left(a_{o s c}\right) k_{1}^{*} \frac{w_{o}^{*}}{Q^{*}} k_{o} w_{o}^{2} \\
& w_{o s c}^{2} A=w_{o}^{2} w_{o}^{* 2}
\end{aligned}
$$

The output of the biological filter (the input to the nonlinear element) is approximately sinusoidal due to the band-pass characteristics of the global structure. This fact allows us to use the linear approximation stated in the describing function method [29] for the mathematical treatment of the nonlinear element. As a result, the transfer function of this nonlinear element can be approximated as $N\left(a_{o s c}\right)=4 V_{r e f} / \pi a_{\text {osc }}$ where $V_{r e f}$ is the comparator voltage reference and $a_{o s c}$ is the amplitude of the voltage oscillation.

We can find an oscillatory solution for each fill factor, ff, which directly correlates the main oscillation parameters with the occupied cell-culture area and the number of cell in the culture. For the case described in Figure 3, Figure 9 illustrates the frequency and amplitude of the predicted oscillations. For every application, the sensitivity of the oscillation parameters with regard to the filter and the comparator has to be determined in order to maximize both the oscillation frequency and the dynamic range. Let us remark how the oscillation frequency increases monotonically in the range $[7560,7920] \mathrm{Hz}\left(0.16 \mathrm{~Hz} / \mu \mathrm{m}^{2}\right.$ of electrode area occupied by cells) and the oscillation amplitude also increases monotonically in the interval $[0,500] \mathrm{mV}$ $\left(0.2 \mathrm{mV} / \mu \mathrm{m}^{2}\right.$ of electrode area occupied by cells), when the cell-electrode area overlap $\left(A_{c}\right)$ is increasing from 0 to 1 . Since the signal level in $V_{\text {out }}$ (see Figure 8) is very small, due to electrode modeling constraints, the secondary output $\left(V_{\text {out } 2}\right.$ in Figure 8$)$ is considered as the potential output voltage, thus improving the dynamic range. 

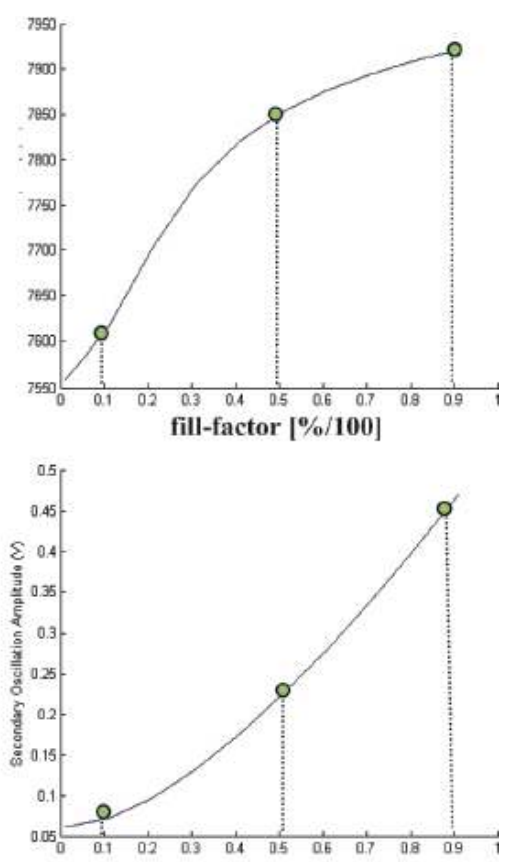

fill-factor $[\% / 100]$

Figure 9. Oscillation parameters obtained from cell-to-electrode area overlap defined by $f f=A / A f f$ in $[0,0.9]$. The approximated sensitivities are $0.16 \mathrm{~Hz} / \mu \mathrm{m}^{2}$ for $f_{\text {osc }}$ and $0.2 \mathrm{mV} / \mu \mathrm{m}^{2}$ for $V_{\text {out } 2^{\prime}}$ using a gold microelectrode of $2500 \mu \mathrm{m}^{2}$ surface.

\subsection{Measurement circuits}

A discrete component OPAMP-based prototype was implemented in the laboratory as a proof of concept, following the scheme proposed in Figure 8 [24]. The circuit is composed upon the following block, depicted in Figure 10:

- A second-order active band-pass filter (BPF). The cutoff frequency that can be tuned in the range of $6.6-13 \mathrm{kHz}$, depending on system sensitivity. Also, $Q$ and $K$ (gain) filter parameters are configurable.

- A voltage comparator with a hysteresis block for noise reduction (COMP).

- A noise reduction block formed by a low-pass filter (LPF) and a high-pass filter (HPF).

- An automated gain control (AGC), required for limiting the voltage amplitude applied to the electrodes. Alternatively, the AGC system can be used to easily extract useful information from the sample values. This block assures that non-harmful voltage level will pass through the sample cells, thus avoiding risk of sample degradation.

- An analog multiplexer circuit controlled by the digital microcontroller, for channel selection. This enables several cell-culture measurements in real time. Our prototype is capable 
of performing measurements in up to 16 cell cultures simultaneously. A digital block containing a microcontroller capable of driving control signals, measuring signals using an integrated ADC, and responsible for communication management of a Bluetooth link. This block also contains some environmental sensor device for sampling additional experiment variables like temperature, humidity, and $\mathrm{CO}_{2}$ concentration.

A more extensive explanation of the circuit design employed can be found at [24]. An image of the prototype implemented is shown in Figure 11. Electronic circuits for BI measurements are inside a specific designed box to be protected for humidity in the incubator.

\subsection{Data processing and wireless communication}

The system functionality relies on several digital devices. As it was depicted in the previous section, our sensor device constrains several blocks, including a digital section based on an ARM Cortex-M7 device. This device has a rich set of several peripheral devices. In our project we will use analog-to-digital converter (ADC) for sampling data and real-time clock (RTC) for synchronization. Cortex-M7 devices implement a floating-point unit (FPU), enabling them for executing signal processing algorithms.

This device is capable of managing the whole sensing task and performs communication through a Bluetooth module, acquiring the signal from the measurement circuit presented. These data are obtained by an ADC integrated in the microcontroller. Further processing is required in order to obtain valuable information from the sampled signals.

As it was already presented in the previous section, the information from which cell-culture monitoring is performed is the signal produced in a oscillator having the cell culture in the signal path. Signals can be observed from the perspective of the time domain and the frequency domain. Fast Fourier transform (FFT) algorithm computes the conversion of data to the frequency domain, providing us with the necessary analysis which will enable the deter-

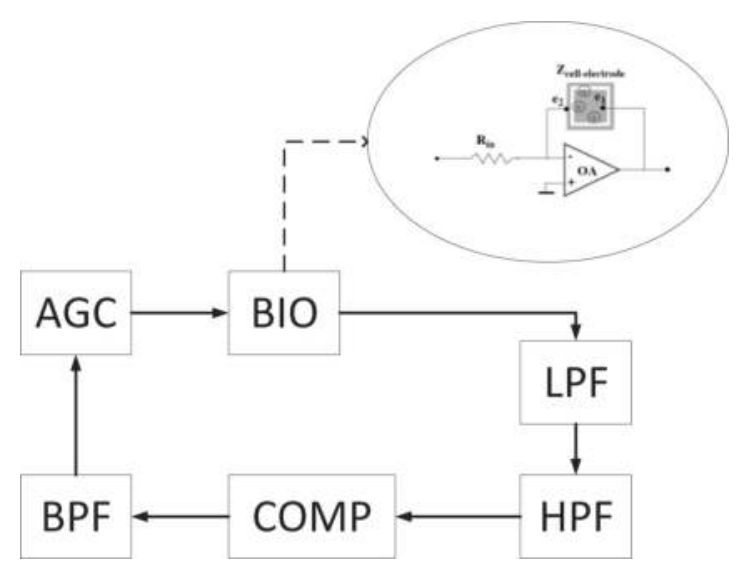

Figure 10. Main circuit blocks for bioimpedance measurement. 


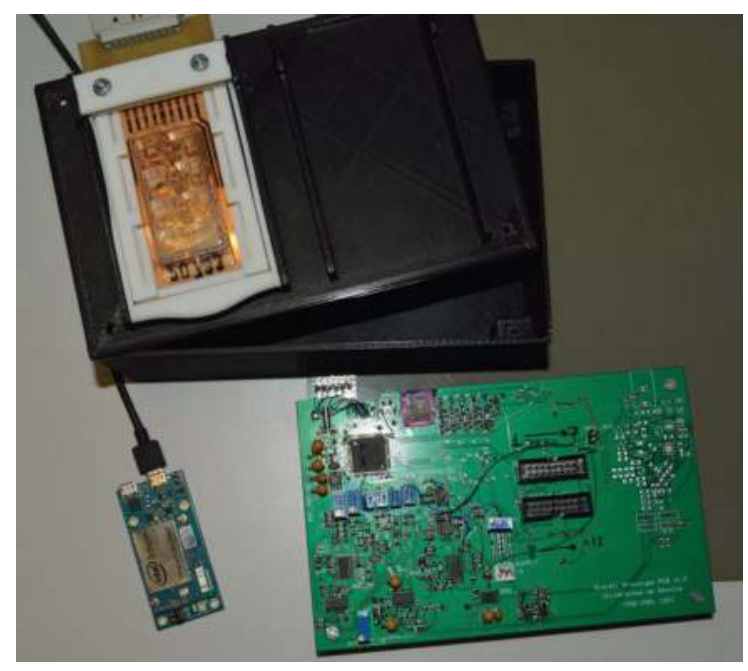

Figure 11. Photograph of the full measurement system including the electrodes for cell culture.

mination of the main frequency in the biological oscillator. The algorithm implementation for this analysis can serve to obtain accurate information. Data derived from this analysis assure less than $1 \%$ deviation from the original signal.

A complete view of the system architecture using the sensor devices was depicted before. The commercial system-on-a-chip (SoC) and the PC dataserver are shown on the scheme at Figure 1.

The whole prototype system is composed of three devices: a PC server installed in the engineering laboratory, a system-on-a-chip (SoC) device in the biomedical laboratory, and the data acquisition system (sensor) inside the cell-culture reactor, for managing the whole experiment. A Bluetooth link exists between the $\mu \mathrm{P}$ and the SoC. The SoC will communicate with the data server by the internet.

The external device is using an Intel Edison platform. This SoC provides a Linux core with Bluetooth and Wi-Fi links. This device is in charge of managing the multiple sensors (S01, S02, S03, S04, etc.) that might be analyzing cell cultures in the biomedical laboratory within Bluetooth range. The system is driving such experiments with a direct communication with the data server over the internet. The experiment configuration is defined in the remote database, and data are stored there after acquisition.

The SoC connects with the remote server which will act as a database storage system and will serve data in a comprehensive manner using a web application directly implemented on it. The experiment control variables (e.g., sample time), can be can defined and modified from the web application. This interface also shows that the data are being gathered from the experiment in real time. The data being depicted in the web application are frequency and amplitude obtained from microelectrodes. The interface is live and online and can be checked in http://jarvis.dte.us.es/mixcell. None of the physical, chemical, or biological factors in the 
experiments are affected by the wireless communication, as it is shown in Section 3. A snapshot showing data obtained from simulation models is on Figure $\mathbf{1 2 .}$

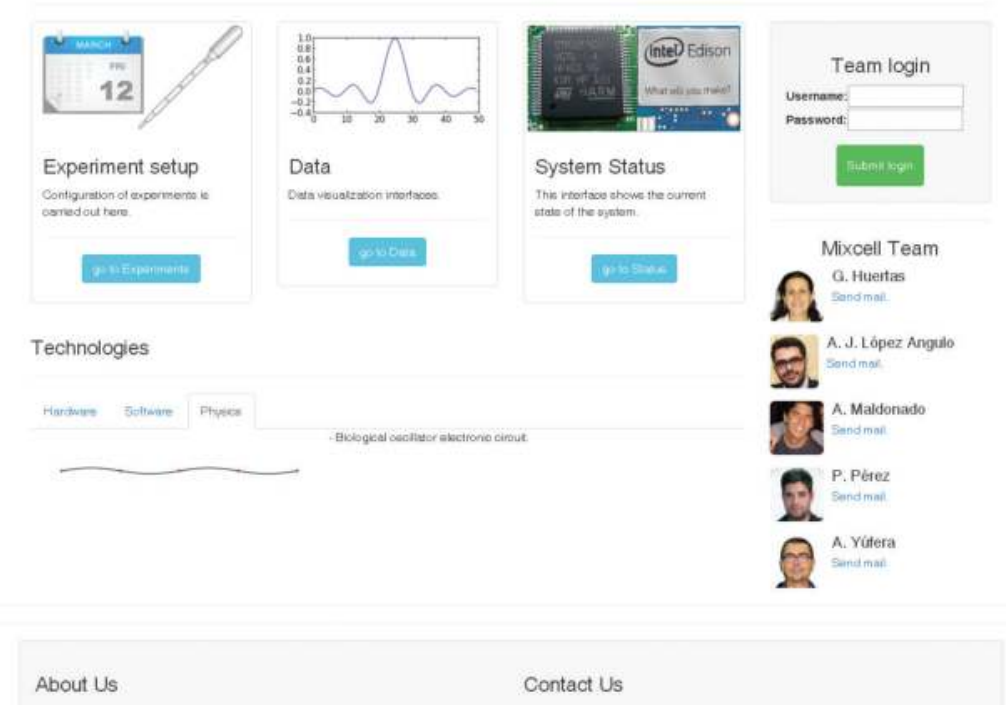

Figure 12. Front end developed for cell-culture control, supervising, and data processing.

This system architecture design is optimized for data integrity and power consumption. The $\mu \mathrm{P}$ system inside the cell-culture incubator chamber is in standby mode most of the time. When the RTC reactivates the whole system, a connection between the $\mu \mathrm{P}$ and the external SoC is established using the Bluetooth link. The $\mu \mathrm{P}$ then samples the amplitude value and calculates the frequency by the means provided by the fast Fourier transform (FFT). After processing the raw data, the device sends the valuable information to the external SoC which will decide according to the experiment parameters stored on the database which will be the next step for the experiment; take another measurement, measure another channel, read additional sensor data, or send the system to standby mode for a while (measurement period). All data recovered by the SoC are stored both, locally and in the remote database in the server. An important point from this architecture is that it can be implemented to support further functionalities (multiple experiments being carried out by the biomedical researcher at the same time using multiple digital acquisition systems inside the reactor). Further improvements would include notifications based on researcher experiment definitions and programming different alarms according to different experiment statuses.

\section{Cell-culture experiments}

Considering the system described in Section 2, Section 3 finally describes how a cell-culture assay test is performed. Specifically, some facilities for testing available from the control web 
page are illustrated in an initial experiment. The access to this front end can be done through the web page www.jarvis.dte.us.es/mixcell.. The validation of the technique and cost-effectiveness of the method are finally presented.

\subsection{Web page functionalities}

Laptops and phone mobiles are expected to be the devices for remote monitoring the cellculture experiments in real time. The current version of the web page is shown in Figure 12. This web page can be used for reporting data of cell-culture evolution, visualization of many experiments (as it works as an experiment database), modification of the test conditions, optimization of the system performance, etc. In particular, some facilities implemented are:

- Creating and defining new experiments

- Checking experiment status

- Modifying active experiments (sampling time, ending time, etc.)

- Checking environment variables (temperature, humidity, $\mathrm{CO}_{2}$ concentration)

- Viewing eight measures (one per well) simultaneously or selecting one or various wells of interest

- Viewing oscillation frequency and amplitude for the bio-oscillator

- Performing different statistical measurements over data

- Exporting data to several formats (.xls, .mat, csv)

- Configuring alerts for the biomedical researchers

- Checking system variables (battery level, control variables)

\subsection{Data assays}

The eight $8 \mathrm{~W} 10 \mathrm{E}$ wells were seeded with cells and medium to test our system. In this way, culture assays with only medium ( 2 wells), plus 500 cells ( 2 wells), 1000 cells ( 2 wells), and 2000 cells (2 wells) were programmed. Observation times were defined at 5 (P1) and 7 (P2) days. The frequency $\left(f_{\text {osc }}\right)$ and amplitude $\left(a_{\text {osc }}\right)$ of the oscillations measured are summarized in Table 2. Figure 13 illustrates the waveforms measured at 5 days for $V_{\text {out } 2}$ and the comparator output at the 500 cells case. $V_{\text {out } 2}$ oscillation amplitude and frequency are $1.30 \mathrm{~V}$ and $7912 \mathrm{~Hz}$, respectively. These values are increased to $1.54 \mathrm{~V}$ and $7922 \mathrm{~Hz}$ at the $2000 \mathrm{cells}$ case (Figure 14). The frequency variation is small in these experimental measurements, due mainly to the inclusion of an AGC, employed to improve the output voltage dynamic range at $V_{\text {out }}$ (Figure 8), to be consistent with the modeling constraints. This block limits the sensibility of the frequency measurements. However, it should be noticed that just amplitude measurements are enough to validate the proposed OB technique. Finally, the amplitude and oscillation frequencies at P1 are represented in Figures 15 and 16, respectively. The direct linear dependence of both signals with the fill factor, validating our system approach, can be observed. 


\begin{tabular}{lllllll}
\hline Well- $\mathbf{n}_{\text {cells }}$ & \multicolumn{5}{c}{ P1: $\mathbf{5}$ days of growth } & \multicolumn{3}{l}{ P2: 7 days of growth } \\
\cline { 2 - 7 } & ff (\%) & $a_{\text {osc }}[\mathbf{V}]$ & $f_{\text {osc }}[\mathbf{k H z}]$ & $\mathbf{f f}$ (\%) & $a_{\text {osc }}[\mathbf{V}]$ & $f_{\text {osc }}[\mathbf{k H z}]$ \\
\hline $1-$ Med & 0 & 0.76 & 7.862 & 0 & 0.76 & 7.860 \\
$2-500$ & 58 & 1.30 & 7.911 & 79.5 & 1.42 & 7.918 \\
$3-1000$ & 72 & 1.62 & 7.924 & 90.5 & 1.66 & 7.937 \\
$4-2000$ & 51 & 1.10 & 7.899 & Death c. & - & - \\
$5-$ Med & 0 & 0.76 & 7.874 & 0 & 0.74 & 7.860 \\
$6-500$ & 62 & 1.38 & 7.911 & 95 & 1.54 & 7.930 \\
$7-1000$ & 48 & 1.18 & 7.899 & 56 & 1.22 & 7.911 \\
$8-2000$ & 80 & 1.54 & 7.924 & 100 & 1.66 & 7.937 \\
\hline
\end{tabular}

Table 2. Electrode surface occupation $(f f)$ in $\%$, amplitude $\left(a_{o s c}\right)$, and frequency $\left(f_{o s c}\right)$ of oscillations obtained from test with living cells.

Comparing these results with previous works [21, 23], none of the physical, chemical, or biological factors in the experiments are affected by the wireless communication, as it was expected. Wireless signals emitted by the reported equipment are similar to those emitted by Wi-Fi servers and cellular phones present at biomedical labs.

\subsection{Cost-effectiveness of the method and implementation possibilities}

The reported technique enables biomedical researchers and lab technicians to continuously perform cell-culture assay supervision, offering a label-free, high-throughput, and high-content screening device for cellular research, avoiding the classical end-point techniques, but also a significant workload and cost material reduction.

This ECIS method can result in massive saving for pharmaceutical and biotechnology companies involved in drug development process. Due to its label-free nature and its fast detection potential, the system can avoid false-positive and exclude dead-end drug candidates from devel-

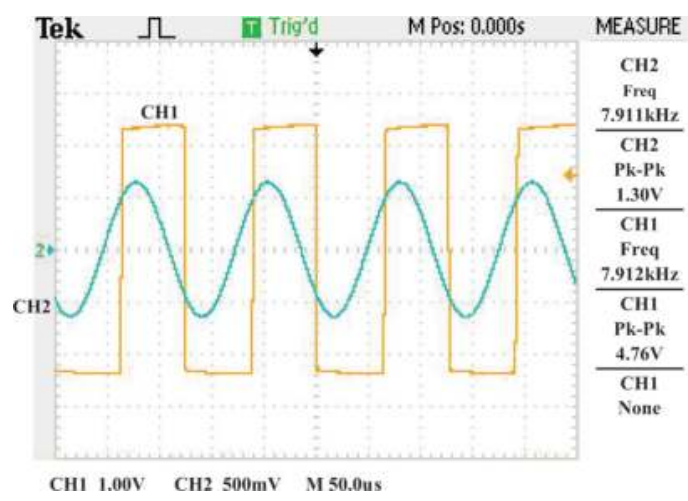

Figure 13. Voltage waveforms of $\mathrm{V}_{\text {out2 }}(\mathrm{CH} 2)$ and comparator output (CH1). 500 cell case, at P1. 


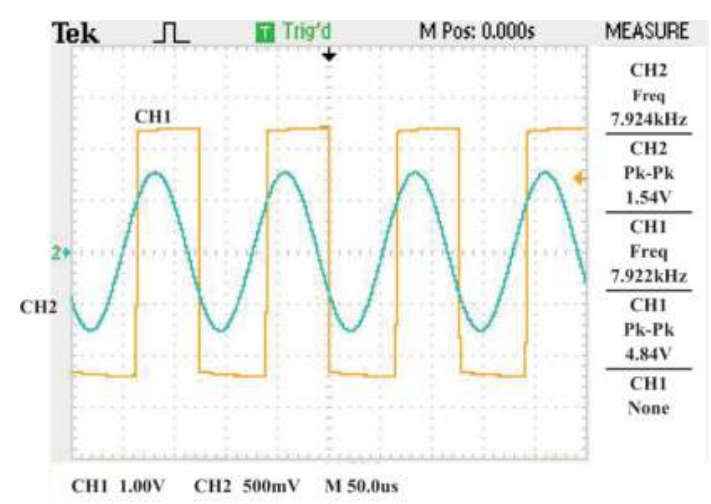

Figure 14. Voltage waveforms of $\mathrm{V}_{\text {out } 2}(\mathrm{CH} 2)$ and comparator output (CH1). 2000 cells case, at P1.

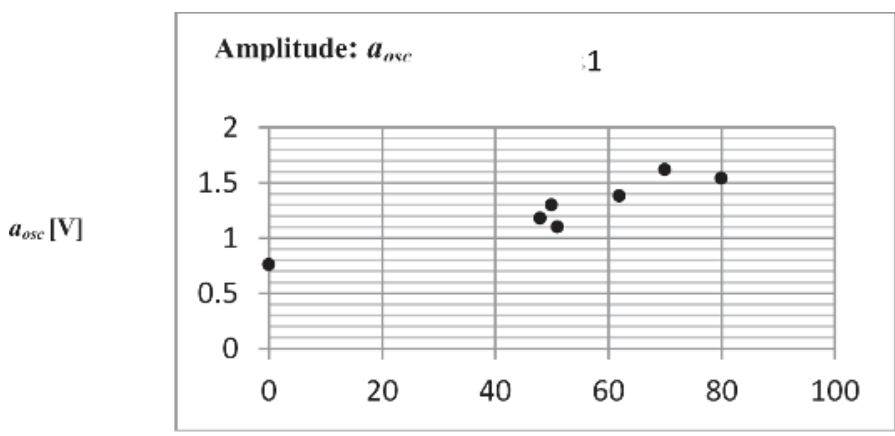

fill-factor $[\%]$

Figure 15. Amplitude versus fill factor (data from 5 days).

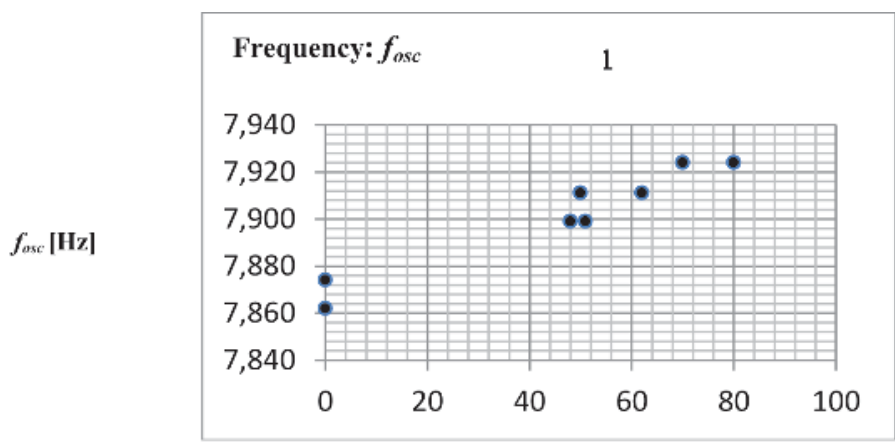

fill-factor $[\%]$

Figure 16. Frequency versus fill factor (data from 5 days). 
opment at an early stage. The ECIS method only requires one culture to perform the full assay, and not many samples, as commonly, for time supervision and characterization of the assays, minimizing the statistical dispersion at growing tests. In spite the higher cost of disposable microelectrodes, in comparison with traditional cell-culture plates, the aforementioned advantages in the automation of the process enable the reduction of the overall costs in laboratories.

Furthermore, the system enables a real-time remote sensing option for the biomedical assays, by using wireless communication protocols and electronic devices for internet access, such as laptops and cellular phones. This facility (remote sensing) is easily implemented once the ECIS signal is acquired and can be easily set in all types of labs, proposing a new concept of cell-culture assays, allowing biomedical researchers to supervise a culture in any time from any place, and making it easy and efficient for lab protocols implementation.

\section{Conclusions}

In this work, we present a system that allows, simultaneously, to measure the status of cellculture assays in real time, and its remote sensing thanks to internet access from laptops or mobile phones. The reported technique offers a label-free, high-throughput, and high-content screening device for cellular research, avoiding the classical end-point techniques, but also a significant workload and cost material reduction. It provides an easy way to analyze current status of cell-culture samples without opening cell-culture oven or performing direct measurements which may affect sample integrity. This measurement process is innocuous for the living cell sample in the culture medium.

To perform the measurements, the work presents a system for cell-culture monitoring using the oscillation-based concept. A simple topology based on a nonlinear element embedded in a feedback loop is employed to convert the cell-culture under test (CCUT) into a suitable bio-oscillator. The technique does not avoid any excitation signal as the standard ECIS technique. The proposed method eliminates the need to carry out a statistical evaluation of multiple samples as it is usual in most ECIS techniques, delivering a deterministic and robust test method for cellculture test characterization. Commercial circuit components have been employed in a practical implementation proposed to prove the concept. Both simulation and experimental results validate the predictions. Actually, the test performance with cell cultures has been demonstrated, deriving on calibration curves for the fill factor parameter that allows a not end-point real-time, low-cost technique for monitoring cell-culture assays. This facility (remote sensing) is easily implemented once the ECIS signal is acquired and can be easily set in all types of laboratories.

\section{Acknowledgements}

This work was supported in part by the Spanish-founded project, TEC 2013-46242-C3-1-P: Integrated Microsystem for Cell Culture Assays, cofinanced with FEDER. Also, we thank the teams of Dr. Pablo Huertas, at the Centro Andaluz de Biologia Molecular y Medicina 
Regenerativa (CABIMER), and Dra. Paula Daza, from Cell Biology Department of the University of Seville, for their help to cell-culture assays and experiment advising.

\section{Author details}

Pablo Pérez ${ }^{1,2}$, Andrés Maldonado-Jacobi ${ }^{1,3}$, Antonio J. López ${ }^{1,3}$, Cristina Martínez ${ }^{1,2}$, Alberto Olmo $^{1,2}$, Gloria Huertas ${ }^{1,3}$ and Alberto Yúfera ${ }^{1,2 *}$

*Address all correspondence to: yufera@imse-cnm.csic.es

1 Microelectronic Institute of Seville, Microelectronic National Center (CNM-CSIC), Spain

2 Department of Electronic Technology, University of Seville, Spain

3 Department of Electronic and Electromagnetism, University of Seville, Spain

\section{References}

[1] Schwan, H. P.: Electrical properties of tissue and cell suspensions. in Advances in Biological and Medical Physics. New York: Academic press. 1957. Vol. 5, pp. 147-224.

[2] Grimnes S. and Martinsen O.: Bioimpedance and Bioelectricity Basics. Second edition. Academic Press, Elsevier (USA). 2008.

[3] Radke S. M. and Alocilja E. C.: Design and fabrication of a microimpedance biosensor for bacterial detection, IEEE Sensor Journal. 2004. Vol. 4, n. 4, pp. 434-440.

[4] Blady B. and Baldetorp B.: Impedance spectra of tumour tissue in comparison with normal tissue; a possible clinical application for electrical impedance tomography. Physiological Measurement. 1996. Vol. 17, suppl. 4A, pp. A105-A115.

[5] Qiao G., Wang W., Duan W., Zheng, F., Sinclair, A. J. and Chatwin, C. R.: Bioimpedance analysis for the characterization of breast cancer cells in suspension. IEEE Transaction on Biomedical Engineering. 2012. Vol. 59, n. 8, pp. 2321-2329.

[6] Giaever I. and Keese Ch.: Use of electric fields to monitor the dynamical aspect of cell behaviour in tissue culture. IEEE Transaction on Biomedical Engineering. 1986. Vol: 33, n. 2. pp. 242-247.

[7] Giaever I. and Keese Ch.: Micromotion of mammalian cells measured electrically. Proceedings of the National Academy of Sciences USA. 1991. Vol. 88, pp. 7896-7900.

[8] Wang P. and Liu Q. editors: Cell-Based Biosensors: Principles and Applications. Artech House Series (USA) 2010.

[9] Daza P., Olmo A., Cañete D. and Yúfera A.: Monitoring living cell assays with bioimpedance sensors. Sensors and Actuators B: Chemical. 2013. Vol. 176, pp. 605-610. 
[10] Müller J., Thirion C., Pfafflal M. W.: Electric cell-substrate impedance sensing (ECIS) based real-time measurement of titer dependent cytotoxicity induced by adenoviral vectors in an IPI-2I cell culture model. Biosensors and Bioelectronics. 2011. 26, pp. 2000-2005.

[11] Linderholm P., Braschler T., Vannod J., Barrandon Y., Brouard M. and Renaud P.: Twodimensional impedance imaging of cell migration and epithelial stratification. Lab Chip. 2006. Vol. 6, n. 9, pp. 1155-1162.

[12] Pradhan R., Mandal M., Mitra A. and Das S.: Assessing cytotoxic effect of ZD6474 on MDA-MB-468 cells using cell-based sensor. IEEE Sensor Journal. 2014. Vol. 14, n. 5, pp. 1476-1481.

[13] Huang X. et al.: Simulation of microelectrode impedance changes due to cell growth. IEEE Sensors Journal. 2004. Vol. 4, n. 5, pp. 576-583.

[14] Borkholder D. A.: Cell-Based Biosensors Using Microelectrodes. PhD Thesis, Stanford University (USA). 1998.

[15] Joye N. et al.: An Electrical Model of the Cell-Electrode Interface for High-density Microelectrode Arrays. 30th Annual International IEEE EMBS Conference. 2008. pp. $559-562$.

[16] Olmo A. and Yúfera A.: Computer Simulation of Microelectrode Based Bio-Impedance Measurements With COMSOL. Third International Conference on Biomedical Electronics and Devices, BIODEVICES. 2010. pp. 178-182. Valencia (Spain) 20-23.

[17] Yúfera A., Gallego E. and Molina J.: ImagCell: a computer tool for cell culture image processing applications in bioimpedance measurements. In Advances in Experimental Medicine and Biology. 2011. Vol. 696, pp. 733-740.

[18] Guermandi M., Cardu R., Franchi Scarselli E. and Guerrieri R.: Active electrode IC for EEG and electrical impedance tomography with continuous monitoring of contact impedance. IEEE Transactions on Biomedical Circuits and Systems. 2015. Vol. 9, n. 1, pp. 21-33.

[19] Ha S., Kim C., Chi Y. M., Akinin A., Maier C., Ueno A. and Cauwenberghs G.: Integrated circuits and electrode interfaces for noninvasive physiological monitoring. IEEE Transaction on Biomedical Engineering. 2014. Vol. 61, n. 5, pp. 1522-1537.

[20] Beach R. D. et al.: Towards a miniature in vivo telemetry monitoring system dynamically configurable as a potentiostat or galvanostat for two- and three- electrode biosensors. IEEE Transaction on Instrumentation and Measurement. 2005, Vol. 54, n. 1, pp. 61-72.

[21] Huertas G., Maldonado A., Yúfera A., Rueda A. and Huertas J. L.: The bio-oscillator: a circuit for cell-culture assays. IEEE Transactions on Circuits and Systems. II Express Briefs. 2015. Vol. 62, n. 2, pp. 164-168.

[22] Wissenwasser H., Vellekoop M. J., and Heer R.: Signal generator for wireless impedance monitoring of microbiological systems. IEEE Transactions on Instrumentation and Measurements. 2011. Vol: 60, n. 6, pp. 2039-2046. 
[23] Huertas G., Maldonado A., Yúfera A., Rueda A. and Huertas J. L.: Oscillation-Based Test Applied to Cell Culture Monitoring. IEEE Sensors Conference. 2013, pp. 842-845. Baltimore, USA.

[24] Maldonado A., Normando J., Huertas G. and Yúfera A.: A Cell-Culture Real-Time Monitoring System. Texas Instruments. First place at "Analog Design Contest in Europe" (www.ti.com/tiic-eu). 2013.

[25] Manickam A., et al.: A CMOS electrochemical impedance spectroscopy (EIS) biosensor array. IEEE Transaction on Biomedical Circuits and Systems. 2010. n. 6, pp. 379-390.

[26] Yúfera A. et al.: A tissue impedance measurement chip for myocardial ischemia detection. IEEE Transaction on Circuits and Systems: Part I. 2005. Vol. 52, n. 12 pp. 2620-2628.

[27] Yúfera A. and Rueda A.: Design of a CMOS closed-loop system with applications to bioimpedance measurements. Microelectronics Journal. Elsevier. 2010. Vol. 41, pp. 231-239.

[28] Huertas G., Vázquez D., Rueda A. and Huertas J. L.: Oscillation-Based Test in MixedSignal Circuits. Springer (The Netherlands). 2006.

[29] Fleischer F. et al.: A switched capacitor oscillator with precision amplitude control and guaranteed start-up. IEEE Journal of Solid-State Circuits. 1985. Vol. 20, n. 2. 
